\title{
HOT-DUST-POOR TYPE 1 ACTIVE GALACTIC NUCLEI IN THE COSMOS SURVEY
}

\author{
Heng Hao ${ }^{1}$, Martin Elvis ${ }^{1}$, Francesca Civano ${ }^{1}$, Giorgio Lanzuisi ${ }^{1,2}$, Marcella Brusa ${ }^{3}$, Elisabeta Lusso ${ }^{4}$, \\ Gianni Zamorani ${ }^{4}$, Andrea Comastri ${ }^{4}$, Angela Bongiorno $^{3}$, Chris D. Impey $^{5}$, Anton M. Koekemoer ${ }^{6}$, Emeric Le Floc' ${ }^{7}{ }^{7}$, \\ Mara Salvato $^{8}$, David Sanders ${ }^{7}$, Jonathan R. TrumP ${ }^{5}$, and Cristian Vignali ${ }^{9}$ \\ ${ }^{1}$ Harvard-Smithsonian Center for Astrophysics, 60 Garden Street, Cambridge, MA 02138, USA; hhao@cfa.harvard.edu, elvis@cfa.harvard.edu \\ 2 INAF-IASF Roma, Via del Fosso del Cavaliere 100, 00133 Roma, Italy \\ ${ }^{3}$ Max Planck Institut für extraterrestrische Physik Giessenbachstrasse 1, D-85748 Garching, Germany \\ ${ }^{4}$ INAF-Osservatorio Astronomico di Bologna, via Ranzani 1, I-40127 Bologna, Italy \\ ${ }^{5}$ Steward Observatory, University of Arizona, 933 North Cherry Avenue, Tucson, AZ 85721, USA \\ ${ }^{6}$ Space Telescope Science Institute, 3700 San Martin Drive, Baltimore, MD 21218, USA \\ ${ }^{7}$ Institute for Astronomy, University of Hawaii, 2680 Woodlawn Drive, Honolulu, HI 96822, USA \\ ${ }^{8}$ IPP-Max-Planck-Institute for Plasma Physics, Boltzmannstrasse 2, D-85748 Garching, Germany \\ ${ }^{9}$ Dipartimento di Astronomia, Università degli Studi di Bologna, via Ranzani 1, I-40127 Bologna, Italy \\ Received 2010 July 8; accepted 2010 October 13; published 2010 October 29
}

\begin{abstract}
We report a sizable class of type 1 active galactic nuclei (AGNs) with unusually weak near-infrared (1-3 $\mu \mathrm{m})$ emission in the $X M M$-COSMOS type 1 AGN sample. The fraction of these "hot-dust-poor" AGNs increases with redshift from $6 \%$ at low redshift $(z<2)$ to $20 \%$ at moderate high redshift $(2<z<3.5)$. There is no clear trend of the fraction with other parameters: bolometric luminosity, Eddington ratio, black hole mass, and X-ray luminosity. The $3 \mu \mathrm{m}$ emission relative to the $1 \mu \mathrm{m}$ emission is a factor of $2-4$ smaller than the typical Elvis et al. AGN spectral energy distribution (SED), which indicates a "torus" covering factor of 2\%-29\%, a factor of 3-40 smaller than required by unified models. The weak hot dust emission seems to expose an extension of the accretion disk continuum in some of the source SEDs. We estimate the outer edge of their accretion disks to lie at $(0.3-2.0) \times 10^{4}$ Schwarzschild radii, $\sim 10-23$ times the gravitational stability radii. Formation scenarios for these sources are discussed.
\end{abstract}

Key words: galaxies: evolution - quasars: general

\section{INTRODUCTION}

Characteristically, active galactic nuclei (AGNs) have hotter dust than starburst galaxies, which has long been employed to select AGNs in near-infrared (NIR) surveys (e.g., Miley et al. 1985; Lacy et al. 2004, 2007; Stern et al. 2005; Donley et al. 2008). In AGNs, dust reaches maximum temperature $(\sim 1000-1900 \mathrm{~K})$ at the smallest equilibrium radius (Barvainis 1987; Suganuma et al. 2006). The hot dust is assigned to the inner edge of the "torus" by the unified model of AGNs (Krolik \& Begelman 1988; Antonucci 1993; Urry \& Padovani 1995). The obscuring torus might be a smooth continuation of the broad-line region (BLR; Elitzur \& Ho 2009), with the BLR extending outward to the inner boundary of the dusty torus (Suganuma et al. 2006).

However, exceptions have been observed and predicted. Observationally, Jiang et al. (2010) found two $z \sim 6$ quasars, without any detectable emission from hot dust, suggesting that these hot-dust-free AGNs are the first generation quasars that do not have enough time to form a dusty torus. Rowan-Robinson et al. (2009) found AGNs with torus to bolometric luminosity ratio of only a few percent.

Emmering et al. (1992) proposed a BLR/torus structure in the disk-wind scenario. The two structures correspond to different regions of a clumpy wind coming off the accretion disk rotating around the black hole (BH). The disk-wind scenario predicts that the torus disappears at luminosities lower than $\sim 10^{42} \mathrm{erg} \mathrm{s}^{-1}$ because the accretion onto the central $\mathrm{BH}$ can no longer sustain the required cloud outflow rate (Elitzur \& Shlosman 2006). In a sample of nearby AGNs, even the BLR disappears at luminosities lower than $5 \times 10^{39}\left(M / 10^{7} M_{\odot}\right)^{2 / 3} \mathrm{erg} \mathrm{s}^{-1}$ (Elitzur \& Ho 2009).
The AGN structure is reflected in the shape of the spectral energy distribution (SED). The maximum dust temperature leads to a characteristic drop in the NIR emission at $1 \mu \mathrm{m}$ (Elvis et al. 1994, E94 hereafter; Glikman et al. 2006). AGNs with weak or no "torus" have an SED with weak or no IR bump, or no $1 \mu \mathrm{m}$ inflection.

We have found a substantial $(\sim 10 \%)$ population of such "hot-dust-poor" (HDP) AGNs by studying the SEDs of 408 $X M M$-COSMOS (Hasinger et al. 2007; Cappelluti et al. 2009) X-ray-selected type 1 (FWHM $>2000 \mathrm{~km} \mathrm{~s}^{-1}$; Elvis et al. 2010) AGNs. All these $X$-ray sources have secure optical and infrared identifications (Brusa et al. 2010) and at least one optical spectrum, from either the Magellan (Trump et al. 2009a), Sloan Digital Sky Survey (SDSS; Schneider et al. 2007), or Very Large Telescope (VLT; Lilly et al. 2007, 2009) surveys.

\section{SELECTION OF HDP OBJECTS}

The HDP AGNs are selected based on the NIR and optical SED shapes. We plot NIR versus optical slopes on either side of $1 \mu \mathrm{m}$ (rest frame, Figure 1; for details see Hao et al. 2010). The $1 \mu \mathrm{m}$ point is not chosen arbitrarily. It is where the blackbody emission of the hot dust at the maximum sublimation temperature (e.g., $1500 \mathrm{~K}$; Barvainis 1987) normally begins to outshine the emission of the accretion disk.

The plot is equivalent to a color-color plot, but utilizes more than four photometric bands. Briefly, we fit power laws $\left(\nu F_{v} \propto v^{\alpha}\right)$ on either side of the $1 \mu \mathrm{m}$ inflection point of the restframe SED: $1 \mu \mathrm{m}-3000 \AA$ to derive an optical slope $\left(\alpha_{\mathrm{OPT}}\right)$ and $3 \mu \mathrm{m}-1 \mu \mathrm{m}$ to derive a NIR slope $\left(\alpha_{\mathrm{NIR}}\right)$. In normal type $1 \mathrm{AGNs}$, these slopes are $\alpha_{\mathrm{OPT}} \sim 1$ and $\alpha_{\mathrm{NIR}} \sim-0.7$ (E94; Richards et al. 2006). In order to obtain slopes that reflect the shape of the SED correctly, we required the number of photometric points of the 


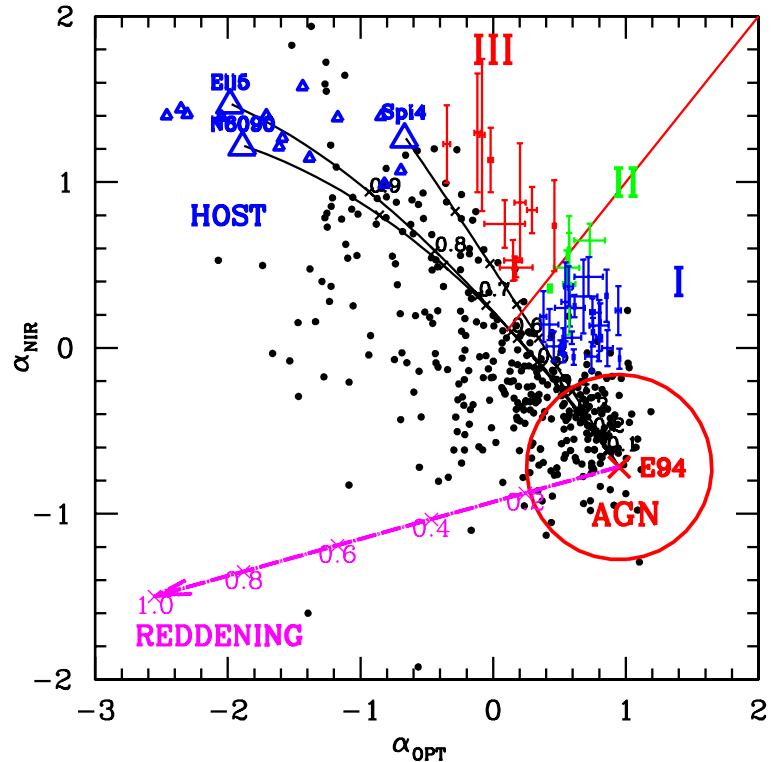

Figure 1. Slope-slope plot of the $X M M$-COSMOS type 1 AGNs. Red cross and red circle show the E94 mean SED and the $1 \sigma$ dispersion of the E94 sources. The blue triangles show different SWIRE galaxy templates. The black lines connecting the E94 and the galaxy templates are mixing curves. The purple line shows the reddening vector of E94. The straight red solid line shows the $\alpha_{\mathrm{OPT}}=\alpha_{\mathrm{NIR}}$ line. Different colors of the points show different class of the HDP AGNs (I, II, and III, see the text for details). The black dots show all the other $X M M$-COSMOS type 1 AGNs.

linear fit to be larger than 2. Out of the 408 X-ray-selected type $1 \mathrm{AGNs}^{10}, 404$ satisfy these criteria. The errors of the slopes are the standard errors of the linear fit.

The $\alpha_{\mathrm{OPT}}-\alpha_{\mathrm{NIR}}$ plot can be conveniently used to separate the nuclear from the host galaxy emission. Figure 1 shows the $\alpha_{\mathrm{OPT}}$ and $\alpha_{\mathrm{NIR}}$ for the E94 radio-quiet AGN template (red cross) and three galaxy templates (a spiral-Spi4, a 5 Gyr old elliptical-Ell5, and a starburst-NGC 6090; big blue triangles) from the "SWIRE Template Library" (Polletta et al. 2007). The black curves show the slopes of the SED templates obtained by mixing the AGN and galaxy with different fractions $(0 \%-100 \%)$ after normalizing the E94 and galaxy templates at $1 \mu \mathrm{m}$. The mixing curves of NGC 6090 and Spi4 define the boundaries of the possible slopes obtained by mixing the E94 with all the 16 available galaxy templates in the SWIRE library (small blue triangles). The magenta line shows the OPT and NIR slopes of the E94 template when reddening $(E(B-V)=0-1 \mathrm{mag})$ is applied. ${ }^{11}$ Reddening primarily affects $\alpha_{\mathrm{OPT}}$.

The majority of the XMM-COSMOS AGNs ( 90\%) are explained by combinations of an AGN, a galaxy, and reddening (Hao et al. 2010), as they lie within the triangular region at the left of the $\alpha_{\mathrm{OPT}}-\alpha_{\mathrm{NIR}}$ plot, bounded by the reddening and mixing curves. However, $\sim 10 \%$ (41 sources) lie in the upper right corner of Figure 1, more than $1 \sigma$ beyond the AGN-host-reddening triangle. These sources have an optical slope consistent with typical AGNs, but a relatively weak IR bump. We name them "hot-dust-poor" (HDP) quasars.

\footnotetext{
10 Three out of the other four (XID $=2119,5320,5617$ ) are low-redshift AGNs with only $K$ band and one IRAC band in the rest-frame NIR SED. The remaining source $(\mathrm{XID}=54439)$ is the highest redshift AGN with only two IRAC bands in the rest-frame NIR SED.

11 We applied the IDL dereddening routine ' FM_UNRED.PRO' ' with the SMC extinction curve (Gordon et al. 2003)
}

\section{HDP SEDS}

There is a range of SED shapes for the 41 HDP AGNs. We further divide these AGNs into three classes, according to their relative positions to the equal slope (red) line in the $\alpha_{\mathrm{OPT}}-\alpha_{\mathrm{NIR}}$ plot. Figure 2 shows examples of SEDs for each class. All wavelengths in this section refer to the rest frame.

Class I. Twenty-four sources lie below the equal slope line (blue symbols in Figure 1). These sources have a normal big blue bump (BBB), but a factor of 2-4 lower than the E94 mean SED at $3 \mu \mathrm{m}$. They all have a rather flat infrared SED shape $\left(\alpha_{\mathrm{NIR}} \sim 0\right)$ extending to at least $10 \mu \mathrm{m}$ (rest frame; Figure 2, left). Seven have high luminosities ( $>5 L_{*}$, ranging from $5.3 L_{*}$ to $24 L_{*}$, where $L_{*}$ is the break point of the galaxy luminosity function; Cirasuolo et al. 2007) at $1 \mu \mathrm{m}$ and hence should have weak host galaxy contribution, while the rest have luminosities $0.76 L_{*}-4 L_{*}$.

Class II. Six sources are consistent with lying on the equal slope line (green symbols in Figure 1). The infrared emission could be the exposed continuation of the BBB to longer wavelengths $(\sim 2-3 \mu \mathrm{m})$. Two out of six have high luminosities ( $>5 L_{*}, 5.4 L_{*}-13 L_{*}$ ) at $1 \mu \mathrm{m}$, while the rest have luminosities $0.32 L_{*}-2.7 L_{*}$.

Class III. Eleven sources lie above the equal slope line (red symbols in Figure 1). These sources have flatter BBB than normal, possibly due to reddening. Some of them show quite strong $\sim 10 \mu \mathrm{m}$ continuum emission (Figure 2 , right). The class III sources have luminosities of $0.53 L_{*}-5.3 L_{*}$.

We calculated the mean SEDs of the HDP AGNs in each class and compared them with the mean SEDs of the other type 1 AGNs in the $X M M$-COSMOS sample having the same range of optical slopes for that class, i.e., normal type 1 AGNs with same BBB shape (Figure 2, upper). The HDP AGNs show relatively weaker $1-3 \mu \mathrm{m}$ emission, by a factor of 1.6 for class I, 3 for class II, and 2.5 for class III.

\section{SOURCE PROPERTIES}

\subsection{Correlations}

We compared a number of observed properties of the HDP AGNs with the whole $X M M$-COSMOS sample: redshift $(z)$, bolometric luminosity ( $\left.L_{\mathrm{bol}}, 24 \mu \mathrm{m}-912 \AA\right)$, BH mass $\left(M_{\mathrm{BH}}\right.$; Trump et al. 2009b; Merloni et al. 2010), Eddington ratio $\left(\lambda_{E}\right.$; Lusso et al. 2010), the optical to X-ray spectral index $\left(\alpha_{\mathrm{ox}}\right),{ }^{12}$ the X-ray luminosity at rest frame $2 \mathrm{keV}\left(L_{2 \mathrm{keV}}\right)$, the X-ray hardness ratio $(\mathrm{HR})^{13}$, and radio loudness $\left(q_{24}\right) .{ }^{14}$ All the values of these parameters were reported in Lusso et al. (2010), Brusa et al. (2010), and Hao et al. (2010). In the HDP sample, only one source $^{15}$ is radio loud (Elvis et al. 2010).

We used the Kolmogorov-Smirnov (K-S) test to compare the distributions of each parameter for the HDP AGNs with the whole sample (HDP AGNs excluded). The K-S probabilities are reported in Table 1 . Only $z$ shows a significant correlation $\left(p_{\mathrm{K}-\mathrm{S}}=0.02 \%\right)$. Distributions of other parameters $\left(M_{\mathrm{BH}}\right.$, $L_{2 \mathrm{keV}}, \log \lambda_{E}$, and $\left.L_{\mathrm{bol}}\right)$ are indistinguishable for the two samples. We will study the X-ray properties of the HDP AGNs in a subsequent paper. We can also see some of these results in

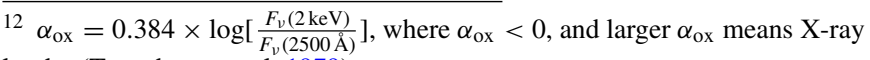
louder (Tananbaum et al. 1979).

$13 \mathrm{HR}=(H-S) /(H+S)$, where $H$ are the $X M M$ counts in the $2-10 \mathrm{keV}$ band and $S$ those in the $0.5-2 \mathrm{keV}$ band (Brusa et al. 2007, 2010).

${ }^{14} q_{24}=\log \left(f_{24} \mu \mathrm{m} / f_{1.4 \mathrm{GHz}}\right)<0$ as radio loud (Appleton et al. 2004).

$15 \mathrm{XID}=167$, class III
} 

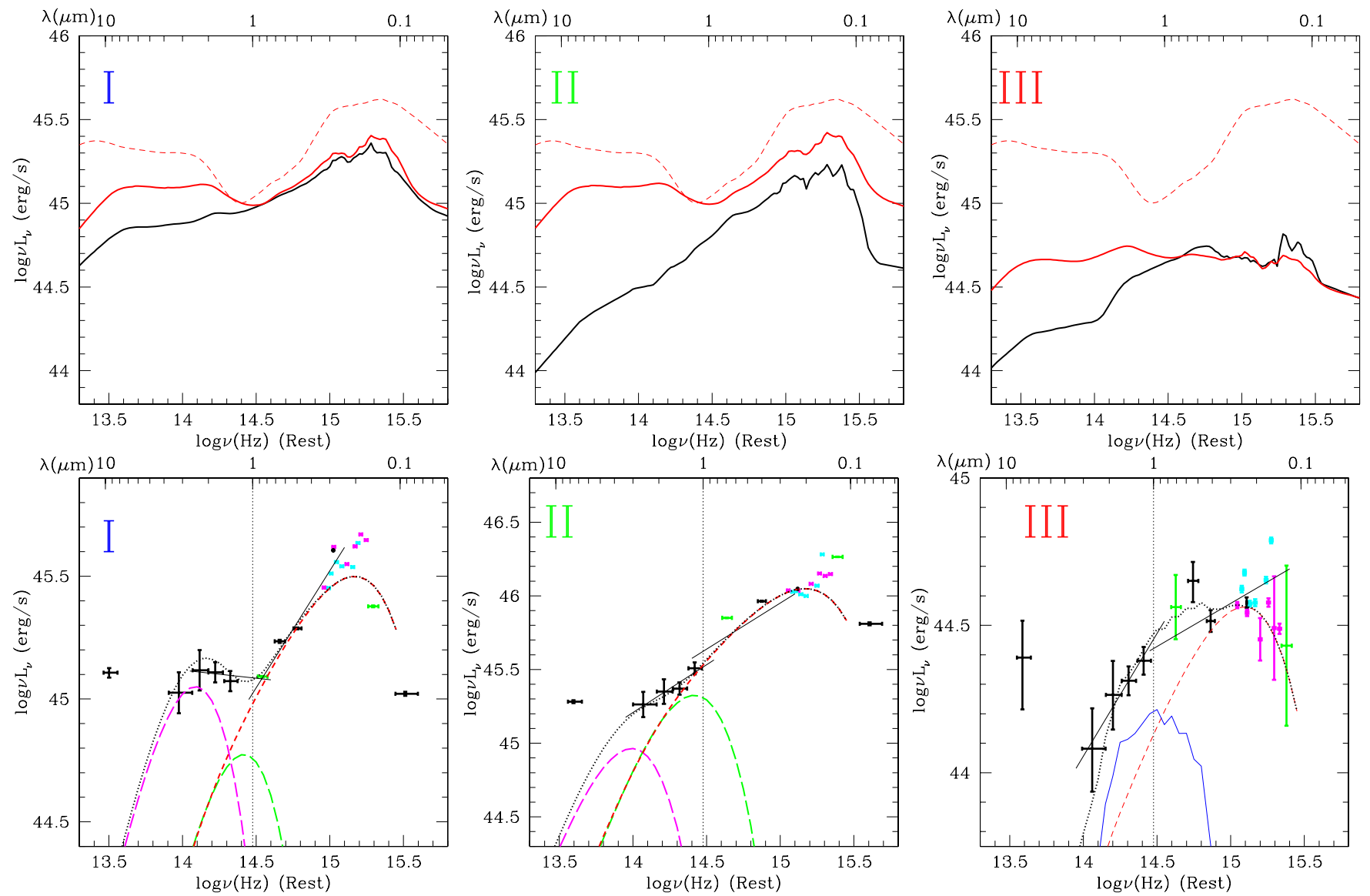

Figure 2. Mean SED and examples of SEDs for each HDP classes: Upper row: mean SED of the three classes of HDP AGNs (black solid line) compared with E94 RQ mean SED (dotted red line) and the mean SED of the normal XMM-COSMOS type 1 AGNs with similar BBB (solid red line). Lower left: class I SED example (XID $=2105$, redshift $z=1.509)$; lower middle: class II SED example (XID $=96, z=2.117)$; lower right: class III SED example (XID $=167, z=2.048$ ). The data points are from Capak et al. (2007), corrected for line emission as in Elvis et al. (2010). The SEDs are fitted with an accretion disk (red dashed line), a dust component (magenta dashed line, single temperature blackbody), and a galaxy component (blue solid line, 5 Gyr elliptical galaxy). The black dotted line shows the SED of the sum of the components. The black straight line shows the power-law fits that give $\alpha_{\mathrm{OPT}}$ and $\alpha_{\mathrm{NIR}}$. The host galaxy contribution is negligible, for XID $=$ 2105 and XID $=96$ at high luminosity $\left(4 L_{*}\right.$ and $13 L_{*}$ at $1 \mu \mathrm{m}$ ). The green dashed line shows the blackbody fitting to outer edge of the accretion disk.

Table 1

K-S Probability

\begin{tabular}{lcc}
\hline \hline Parameter & $N(\mathrm{HDP}) / N(\text { total HDP })^{\mathrm{a}}$ & $p_{\mathrm{K}-\mathrm{S}}$ \\
\hline$z$ & $41 / 363$ & 0.0002 \\
$\mathrm{HR}$ & $41 / 363$ & 0.080 \\
$\log \lambda_{E}$ & $16 / 206$ & 0.166 \\
$\alpha_{\mathrm{Ox}}$ & $41 / 363$ & 0.201 \\
$L_{2 \mathrm{keV}}$ & $41 / 363$ & 0.202 \\
$M_{\mathrm{BH}}$ & $16 / 206$ & 0.630 \\
$L_{\mathrm{bol}}$ & $41 / 363$ & 0.729 \\
\hline
\end{tabular}

Note. ${ }^{\text {a }}$ The number of sources that have the detection of the physical parameter in the format of (HDP AGN)/(whole type 1 AGN sample exclude the HDP AGN).

the fraction plot in Figure 3, where we divided two parameters ${ }^{16}$ into nine bins and plotted the fraction of HDP AGNs in each bin.

The fraction of the HDP AGNs clearly increases with $z$. This fraction is $5.9 \% \pm 0.2 \%$ at $z<2$ but increases to $20.4 \% \pm 0.1 \%$ at $2<z<3.5$. Beyond $z=3.5$, the sample is too small (one source in two bins) to give a useful constraint. A linear regression
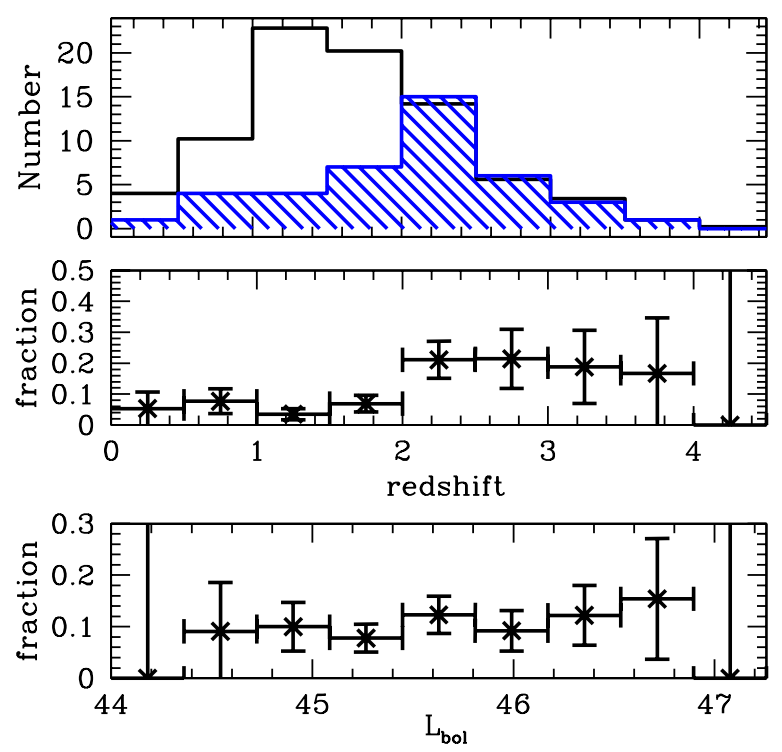

Figure 3. Distribution in the redshift space (top) and fraction of the HDP in the whole sample as a function of redshift (middle) and $L_{\text {bol }}$ (bottom). The black (number divided by 5) and blue histograms show the distribution of all the AGNs and the HDP, respectively.

16 One $(z)$ with the lowest $p_{\mathrm{K}-\mathrm{S}}$ and one $\left(L_{\mathrm{bol}}\right)$ with the highest $p_{\mathrm{K}-\mathrm{S}}$. 
fit of the fractions gives $f_{\mathrm{HDP}}=(0.031 \pm 0.002)(1+z)^{(1.2 \pm 0.1)}$. The photometry used in the SED comes from a point-source correction to the $3^{\prime \prime}$ aperture photometry. Low-redshift SEDs therefore include less host compared to the high redshift ones. This effect will only move the source along the mixing curve, so it cannot explain the observed HDP fraction increase versus redshift.

\subsection{SED Fitting and Physical Parameters}

Studies of NIR spectra of quasars showed that a blackbody spectrum is needed to fit the observed spectrum (Glikman et al. 2006). The BBB is mainly caused by the emission of the accretion disk (e.g., Elvis et al. 1994) and could be reddened by the dust in the host (e.g., Hopkins et al. 2004). At $\sim 1 \mu \mathrm{m}$, the host galaxy has the maximum contribution to the observed type 1 AGN SED (e.g., Polletta et al. 2007). Considering all these effects, we fit all the HDP AGN observed NIR-optical SEDs with three components. (1) Accretion disk emission: we use the standard $\alpha$-disk model in Schwarzschild geometry with electron scattering and Comptonization of soft photons in the disk atmosphere (Siemiginowska et al. 1995), with measured $\mathrm{BH}$ mass and accretion rate where available (Hao et al. 2010) and $E(B-V)=0.1-0.2$ in eight cases to get good chi-square in the UV. (2) Hot dust emission: we use a single temperature blackbody spectrum. (3) Host galaxy emission: we use a $5 \mathrm{Gyr}$ old elliptical galaxy (Polletta et al. 2007). Examples of the fit results are shown in the lower panel of Figure 2.

\subsubsection{Covering Factor}

The NIR SEDs are fitted with a blackbody to get the maximum dust temperature $\left(T_{d}\right)$. The sublimation temperature for graphite and silicate grains are $\sim 1500-1900 \mathrm{~K}$ and $\sim 1000-1400 \mathrm{~K}$, respectively (e.g., Salpeter 1977; Laor \& Draine 1993). $T_{d}$ ranges from $800 \mathrm{~K}$ to $1900 \mathrm{~K}$. Most (19 out of 34) HDP AGNs with dust component have $T_{d}>1400 \mathrm{~K}$, suggesting graphite grains.

Dust can only exist at a radius beyond the dust evaporation radius, given by $r_{d}=1.3 L_{\mathrm{uv}, 46}^{1 / 2} T_{1500}^{-2.8} \mathrm{pc}$ (Barvainis 1987), where $L_{\mathrm{uv}, 46}$ is the total ultraviolet $(1 \mu \mathrm{m}-912 \AA)$ luminosity in units of $10^{46} \mathrm{erg} \mathrm{s}^{-1}$ and $T_{1500}$ is the maximum dust temperature in units of $1500 \mathrm{~K}$. We find that $r_{d}$ ranges from $0.2 \mathrm{pc}$ to $3.9 \mathrm{pc}$ for the HDP sources. The emission area $\left(A_{d}\right)$ is given from the blackbody fitting, and $A_{d}$ ranges from $0.1 \mathrm{pc}^{2}$ to $10.68 \mathrm{pc}^{2}$. The covering factor of the dust component is then $f_{c}=A_{d} /\left(4 \pi r_{d}^{2}\right)$. For example, the results for source XID $=2105$ (Figure 2, left) are $T_{d}=1500 \mathrm{~K}$ and $A_{d}=1.76 \mathrm{pc}^{2}$. The evaporation radius is $0.83 \mathrm{pc}$, corresponding to $f_{c}=20 \%$. $f_{c}$ ranges from $1.9 \%$ to $29 \%$, which are a factor of 2-40 smaller than the $75 \%$ expected from the unified models to give the observed type 2 to type 1 ratio (e.g., Krolik \& Begelman 1988). Even if considering the dependence of type 2 to type 1 ratio on X-ray luminosity (e.g., Gilli et al. 2007), the $f_{c}$ of HDP AGN is still smaller than the $50 \%$ expected for the X-ray bright AGNs.

The above innermost radius estimation is comparable to those reported in Kishimoto et al. (2007, 2009). Kishimoto et al. (2007) also showed that the innermost radii measured by nearIR reverberation are systematically smaller by a factor of $\sim 3$ than the value predicted with the Barvainis (1987) formula. For the HDP AGNs, if the innermost radius of the hot dust is really a factor of 3 smaller, the covering factor would be a factor of 9 larger than the values we calculated, which could reach the $75 \%$ required by the unified models (e.g., Krolik \& Begelman 1988) for most (29 out of 34 ) of the HDP sources.

\subsubsection{Disk Outer Radius}

For 11 HDP AGNs with little host galaxy contamination (defined as $<50 \%$ at $1 \mu \mathrm{m}$ ) and available $M_{\mathrm{BH}}$ and $\lambda_{E}$ (Lusso et al. 2010; Trump et al. 2009b; Merloni et al. 2010), the lack of hot dust emission allows us to see what appears to be the accretion disk emission extending further out well beyond $1 \mu \mathrm{m}$.

We fitted the outer edge of the accretion disk component (red dashed line in Figure 2) with a single temperature $\left(T_{c}\right)$ blackbody spectrum (green dashed line). The outer radius of the accretion disk is (Frank et al. 2002)

$$
R_{\text {out }}=1.1 \times 10^{4} T_{c}^{-\frac{4}{3}} \alpha^{-\frac{4}{15}} \eta^{-\frac{2}{5}} M_{8}^{\frac{11}{15}} \lambda_{E}^{\frac{2}{5}} f^{\frac{8}{5}} \mathrm{pc},
$$

where $M_{8}=M /\left(10^{8} M_{\odot}\right)$ and $f=\left[1-\left(\frac{6 G M}{R c^{2}}\right)^{\frac{1}{2}}\right]^{\frac{1}{4}}$. We assume $\alpha=0.1, \eta=0.1$.

An example is the source XID $=96$ shown in Figure 2 (lower middle; $\left.M_{8}=3.55, \lambda_{E}=0.71\right)$, the blackbody temperature at the outer edge is $3200 \mathrm{~K}$. This gives $R_{\text {out }}=0.47 \mathrm{pc}, \sim 1.3 \times 10^{4}$ Schwarzschild radius $\left(r_{s}\right), 13.6$ times the gravitational stability radius of the accretion disk (Goodman 2003; i.e., the radius beyond which the disk is unstable to self-gravity and should break up). For the $11 \mathrm{HDP}$ AGNs, the $T_{c}$ range from $2200 \mathrm{~K}$ to $4500 \mathrm{~K}$. The $R_{\text {out }}$ range from 0.09 pc to $0.99 \mathrm{pc}$, corresponding to $(0.29-2) \times 10^{4} r_{s}$. These $R_{\text {out }}$ are $\sim 10-23$ times larger than the gravitational stability radius.

\section{DISCUSSION AND CONCLUSIONS}

In a sample of $404 X M M$-selected type 1 AGNs (excluding four with incomplete NIR photometry), we found that $10.1 \% \pm$ $1.7 \%$ have weak NIR emission, indicating a relative paucity of hot dust emission. We call these HDP AGNs.

HDP AGNs have not been reported from SDSS or earlier samples. We have made an initial check of where the Richards et al. (2006) and E94 samples lie on the $\alpha_{\mathrm{OPT}}-\alpha_{\mathrm{NIR}}$ plane. We find similar fraction of HDP AGNs and will report on this study in detail in a later paper. The $\alpha_{\mathrm{OPT}}-\alpha_{\mathrm{NIR}}$ plane is a useful tool for locating outliers.

The fraction of HDP AGNs clearly increases to redshift $z=$ $3.5\left(f_{\mathrm{HDP}}=(0.031 \pm 0.002)(1+z)^{(1.2 \pm 0.1)}\right)$. No trends of the fraction of HDP AGNs with other parameters, notably $L_{\mathrm{bol}}$, are observed.

We divided these HDP AGNs into three classes according to their $\alpha_{\mathrm{OPT}}$ and $\alpha_{\mathrm{NIR}}$. We fitted the HDP SEDs with three components: the accretion disk, a blackbody to represent the hot dust, and the host galaxy. We found that the dust covering factors are $1.9 \%-29 \%$, well below the typical $75 \%$ required by unified models (e.g., Krolik \& Begelman 1988). For the 11 HDP AGNs with little host contribution $\left(<50 \%\right.$ at $1 \mu \mathrm{m}$ ) and available $M_{\mathrm{BH}}$ and $\lambda_{E}$, we estimated the lower limit of the accretion disk's outer radius to be $(0.29-2) \times 10^{4} r_{s}(0.09-0.99 \mathrm{pc})$, corresponding to 10-23 times the gravitational stability radius of an $\alpha$ disk. How these disks stabilized is an open question, or the long wavelength turndown is not $R_{\text {out }}$ as we assumed. These results agree with the NIR disk spectrum uncovered using polarized light in Kishimoto et al. (2008). We ignore further discussion of the sources with strong host galaxy contribution as the system is more complicated to include the also unknown galaxy part.

There are several possibilities to explain the lack of NIR emission in HDP sources. First, most XMM-COSMOS HDPs are 
at $1.5<z<3$ when the universe is $2.1-4.2$ Gyr old, and they have 1-3 Gyr from reionization to form a torus. The Jiang et al. (2010) proposed explanation thus seems unlikely for the XMMCOSMOS HDP AGNs. Second, the luminosity and Eddington ratio distributions of the HDP AGNs and the normal XMMCOSMOS type 1 AGNs are indistinguishable. This rules out the possibility that HDP AGNs are low-luminosity or low-accretion rate sources, unable to support a dusty torus (Elitzur \& Ho 2009). Third, evolutionary scenarios (e.g., Hopkins et al. 2008) predict short-lived Eddington limited outbursts after a merger, which could destroy the innermost dust. The HDP AGNs might then be quasars that had insufficient time to re-form dust in the inner region. Fourth, alternatively, when two galaxies merge, the chaotic dynamics might destroy the "torus." The "torus" then re-forms in a timescale that might allow $10 \%$ of quasars to be HDP. The dust formation timescale depends strongly on pressure and temperature (Whittet 2003; Krügel 2003) making estimates unreliable as both quantities are poorly known. Last, Guedes et al. (2010) suggested that a BH ejected by gravitational wave recoil and carrying along its accretion disk and broad emission line region, but not the hot dust "torus," would appear as an HDP AGN.

The unusual SEDs of HDP AGNs can affect estimates of galaxy bulge and $\mathrm{BH}$ masses derived from SED fitting assuming the BH SED to be the E94 mean SED. Merloni et al. (2010) recently used this method to show that $z \sim 1.5$ quasars deviate from the local $M-\sigma$ relationship (Häring \& Rix 2004). Three of the Merloni et al. (2010) quasars belong to our HDP sample. For these sources, the SED fitting using the E94 template gives a small AGN contribution. Allowing an HDP SED to fit would increase the BH mass and decrease the stellar mass, making them deviate even more from the local $M-\sigma$ relationship. New AGN templates with variable dust bump strengths are needed to derive accurate galaxy and $\mathrm{BH}$ masses in these objects.

The properties of the HDP AGNs still need to be investigated to understand their formation scenario and put them in the context of galaxy and SMBH evolution. In particular, we will check the existing Hubble images and optical and X-ray spectra of HDP AGNs in future papers.

H.H. thanks Sumin Tang for useful discussions. This work was supported in part by NASA Chandra grant number GO7-8136A (H.H., F.C., and M.E.), and by NASA contract NAS8-39073 (Chandra X-ray Center). In Italy this work is supported by ASI/INAF grants I/023/05.

\section{REFERENCES}

Antonucci, R. 1993, ARA\&A, 31, 473

Appleton, P. N., et al. 2004, ApJS, 154, 147
Barvainis, R. 1987, ApJ, 320, 537

Brusa, M., et al. 2007, ApJS, 172, 353

Brusa, M., et al. 2010, ApJ, 716, 348

Capak, P., et al. 2007, ApJS, 172, 99

Cappelluti, N., et al. 2009, A\&A, 497, 635

Cirasuolo, M., et al. 2007, MNRAS, 380, 585

Donley, J. L., Rieke, G. H., Pérez-González, P. G., \& Barro, G. 2008, ApJ, 687, 111

Elitzur, M., \& Ho, L. C. 2009, ApJ, 701, L91

Elitzur, M., \& Shlosman, I. 2006, ApJ, 648, L101

Elvis, M., et al. 1994, ApJS, 95, 1

Elvis, M., et al. 2010, ApJ, submitted

Emmering, R. T., Blandford, R. D., \& Shlosman, I. 1992, ApJ, 385, 460

Frank, J., King, A., \& Raine, D. 2002, Accretion Power in Astrophysics (Cambridge: Cambridge Univ. Press)

Gilli, R., Comastri, A., \& Hasinger, G. 2007, A\&A, 463, 79

Glikman, E., Helfand, D. J., \& White, R. L. 2006, ApJ, 640, 579

Goodman, J. 2003, MNRAS, 339, 937

Gordon, K. D., Clayton, G. C., Misselt, K. A., Landolt, A. U., \& Wolff, M. J. 2003, ApJ, 594, 279

Guedes, J., Madau, P., Mayer, L., \& Callegari, S. 2010, ApJ, submitted (arXiv:1008.2023)

Hao, H., et al. 2010, ApJ, submitted

Häring, N., \& Rix, H.-W. 2004, ApJ, 604, L89

Hasinger, G., et al. 2007, ApJS, 172, 29

Hopkins, P. F., Hernquist, L., Cox, T. J., \& Kereš, D. 2008, ApJS, 175, 356

Hopkins, P. F., et al. 2004, AJ, 128, 1112

Jiang, L., et al. 2010, Nature, 464, 380

Kishimoto, M., Antonucci, R., Blaes, O., Lawrence, A., Boisson, C., Albrecht, M., \& Leipski, C. 2008, Nature, 454, 492

Kishimoto, M., Hönig, S. F., Antonucci, R., Kotani, T., Barvainis, R., Tristram, K. R. W., \& Weigelt, G. 2009, A\&A, 507, L57

Kishimoto, M., Hönig, S. F., Beckert, T., \& Weigelt, G. 2007, A\&A, 476, 713

Krolik, J. H., \& Begelman, M. C. 1988, ApJ, 329, 702

Krügel, E. 2003, The Physics of Interstellar Dust (Bristol: Institute of Physics Publishing)

Lacy, M., Petric, A. O., Sajina, A., Canalizo, G., Storrie-Lombardi, L. J., Armus, L., Fadda, D., \& Marleau, F. R. 2007, AJ, 133, 186

Lacy, M., et al. 2004, ApJS, 154, 166

Laor, A., \& Draine, B. T. 1993, ApJ, 402, 441

Lilly, S. J., et al. 2007, ApJS, 172, 70

Lilly, S. J., et al. 2009, ApJS, 184, 218

Lusso, E., et al. 2010, A\&A, 512, 34

Merloni, A., et al. 2010, ApJ, 708, 137

Miley, G. K., Neugebauer, G., \& Soifer, B. T. 1985, ApJ, 293, 11

Polletta, M., et al. 2007, ApJ, 663, 81

Richards, G., et al. 2006, ApJS, 166, 470

Rowan-Robinson, M., Valtchanov, I., \& Nandra, K. 2009, MNRAS, 397, 1326

Salpeter, E. E. 1977, ARA\&A, 15, 267

Schneider, D. P., et al. 2007, AJ, 130, 367

Siemiginowska, A., Kuhn, O., Elvis, M., Fiore, F., McDowell, J., \& Wilkes, B. J. 1995, ApJ, 454, 77

Stern, D., et al. 2005, ApJ, 631, 163

Suganuma, M., et al. 2006, ApJ, 639, 46

Tananbaum, H., et al. 1979, ApJ, 234, 9

Trump, J. R., et al. 2009a, ApJ, 696, 1195

Trump, J. R., et al. 2009b, ApJ, 700, 49

Urry, C., \& Padovani, P. 1995, PASP, 107, 803

Whittet, D. C. B. 2003, Dust in the Galactic Environment (Bristol: Institute of Physics Publishing) 